\title{
Validity Assessment of 5 Day Repeated Forced- Swim Stress to Model Human Depression in Young-Adult C57BL/6J and BALB/cJ Mice
}

\author{
(D) Joram D. Mul, ${ }^{1}$ Jia Zheng, ${ }^{1}$ and Laurie J. Goodyear ${ }^{1,2}$
}

DOI:http://dx.doi.org/10.1523/ENEURO.0201-16.2016

${ }^{1}$ Section on Integrative Physiology and Metabolism, Joslin Diabetes Center, Harvard Medical School, Boston, Massachusetts 02215, and 'Department of Medicine, Brigham, and Women's Hospital, Harvard Medical School, Boston, Massachusetts 02215

\section{Visual Abstract}

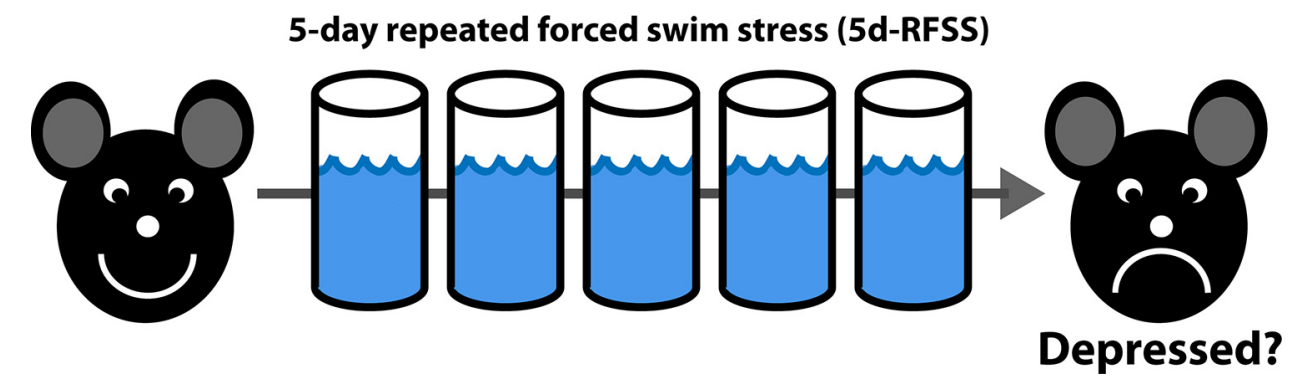

The development of animal models with construct, face, and predictive validity to accurately model human depression has been a major challenge. One proposed rodent model is the $5 \mathrm{~d}$ repeated forced swim stress (5d-RFSS) paradigm, which progressively increases floating during individual swim sessions. The onset and persistence of this floating behavior has been anthropomorphically characterized as a measure of depression. This interpretation has been under debate because a progressive increase in floating over time may reflect an adaptive learned behavioral response promoting survival, and not depression (Molendijk and de Kloet, 2015). To assess construct and face validity, we applied 5d-RFSS to C57BL/6J and BALB/cJ mice, two mouse strains commonly used in neuropsychiatric research, and measured a combination of emotional, homeostatic, and psychomotor symptoms indicative of a depressive-like state. We also compared the efficacy of $5 d-R F S S$ and chronic social defeat stress (CSDS), a validated depression model, to induce a depressive-like state in C57BL/6J mice. In both strains, 5d-RFSS progressively increased floating behavior that persisted for at least 4 weeks. $5 d-R F S S$ did not alter sucrose preference, body weight, appetite, locomotor activity, anxiety-like behavior, or

\section{Significance Statement}

The development of valid animal models to model human depression has been a major challenge. One protocol that has been widely used for its presumptive effects to cause depression in mice is the $5 \mathrm{~d}$ repeated forced swim stress (5d-RFSS) paradigm. 5d-RFSS increases floating behavior during consecutive sessions, but whether this is depressive-like behavior or an adaptive response underlying survival is not clear. We subjected two mouse strains (C57BL/6J, BALB/cJ) to 5d-RFSS followed by a battery of reward-related, homeostatic, and behavioral tests. 5d-RFSS increased floating behavior over time but, importantly, did not induce emotional, homeostatic, or psychomotor symptoms. These findings suggest that $5 d-R F S S$ has no construct or face validity to model human depression in two mouse strains commonly used in neuropsychiatric research. 
immobility behavior during a tail-suspension test compared with nonstressed controls. In contrast, CSDS altered several of these parameters, suggesting a depressive-like state. Finally, predictive validity was assessed using voluntary wheel running (VWR), a known antidepressant intervention. Four weeks of VWR after 5d-RFSS normalized floating behavior toward nonstressed levels. These observations suggest that $5 \mathrm{~d}-\mathrm{RFSS}$ has no construct or face validity but might have predictive validity to model human depression.

Key words: anhedonia; animal model; depression; forced swimming; stress; voluntary wheel running

\section{Introduction}

Major depressive disorder affects approximately one in six individuals during their lifetime and has an enormous social and financial impact on modern society (Greenberg et al., 2003; Kessler et al., 2005). This psychiatric disorder is diagnosed based on symptoms with considerable heterogeneity and without known highly penetrant genetic causes (Krishnan and Nestler, 2008). There is a general need for animal models to study the pathophysiology of depression and identify therapeutic interventions. However, the development of animal models of depression with construct, face, and predictive validity has been a major challenge (Nestler and Hyman, 2010; Belzung and Lemoine, 2011). Nevertheless, several ethologically valid rodent models of depression have been developed and validated, including chronic unpredictable stress (CUS) and chronic social defeat stress (CSDS) (Willner, 2005; Nestler and Hyman, 2010). Despite the validity of these animal models, lack of ethical approval or other limitations has stimulated investigators to keep exploring additional rodent paradigms that model human depression.

One proposed animal model is the $5 \mathrm{~d}$ repeated forced swim stress (5d-RFSS) paradigm, during which mice are forced to swim in a beaker filled with water for 10 min over 5 consecutive days. Several studies have reported a progressive increase in floating during consecutive forced swimming sessions that was maintained for at least 4 weeks, and this behavior is commonly interpreted as the onset and persistence of a depressive-like state (Stone

Received July 12, 2016; accepted October 28, 2016; First published December 12, 2016.

The authors declare no competing financial interests.

Author contributions: J.D.M. and L.J.G. designed research; J.D.M. and J.Z. performed research; J.D.M. analyzed data; J.D.M., J.Z., and L.J.G. wrote the paper.

This work was supported by Department of Health and Human Services/ National Institutes of Health/National Institute for Health Research Grants R01-DK-099511 and R01-DK-101043 (to L.J.G.) and 5P30-DK-36836 (to Diabetes and Endocrinology Research Center, Joslin Diabetes Center). J.D.M. was supported by a mentor-based fellowship (7-08-MN-21) awarded to L.J.G. from the American Diabetes Association.

J.D. Mul's present address: Department of Endocrinology and Metabolism, Academic Medical Center, University of Amsterdam, Postbus 22660, 1100 AD, Amsterdam, The Netherlands, and Netherlands Institute for Neuroscience, Institute of the Royal Netherlands Academy of Arts and Sciences, Meibergdreef 47, 1105 BA, Amsterdam, The Netherlands.

Correspondence should be addressed to Joram D. Mul, Department of Endocrinology and Metabolism, Academic Medical Center, University of Amsterdam, Postbus 22660, 1100 AD, Amsterdam, The Netherlands. E-mail: joramdmul@gmail.com.

DOI:http://dx.doi.org/10.1523/ENEURO.0201-16.2016

Copyright $\odot 2016$ Mul et al.

This is an open-access article distributed under the terms of the Creative Commons Attribution 4.0 International, which permits unrestricted use, distribution and reproduction in any medium provided that the original work is properly attributed. and Lin, 2011; Sun et al., 2011; Serchov et al., 2015). This interpretation has been debated, as increased floating behavior during repeated forced swimming sessions may rather reflect an adaptive learned behavioral response underlying survival (Molendijk and de Kloet, 2015). It has been suggested that to provide more definitive evidence on the presence of a depressive-like state, a combination of emotional symptoms (anhedonia), homeostatic symptoms (sleep, appetite, body weight), psychomotor symptoms (locomotor activity, immobility- and anxiety-like behavior), or direct assessment of the reward circuitry of the brain should be measured (Nestler and Hyman, 2010). Emotional, homeostatic, and psychomotor symptoms are hallmarks of human depression and are important parameters that can be measured objectively in rodents (Nestler and Hyman, 2010).

$5 d-R F S S$ in mice was recently shown to decrease sucrose preference immediately following 5d-RFSS, suggesting anhedonia, and this emotional symptom persisted for at least 4 weeks (Serchov et al., 2015). Maintenance of anhedonia allows for a time window to test and study therapeutic interventions. Here we determined the validity of $5 d$-RFSS to model human depression using a battery of tests that assessed emotional, homeostatic, and psychomotor symptoms. In other words, does 5d-RFSS induce a depressive-like state in mice? For this purpose, we applied a similar $5 d-R F S S$ protocol, as used by Serchov et al. (2015), to two inbred strains commonly used in neuropsychiatric research: young-adult, male, stressresilient C57BL/6J mice and stress-susceptible BALB/cJ mice (Pothion et al., 2004; Farley et al., 2010; Razzoli et al., 2011; Serchov et al., 2015). To thoroughly assess the onset of a depressive-like state, we measured sucrose preference, body weight, food intake, locomotor activity, anxiety-like behavior, and immobility behavior during a tail-suspension test (TST) before and after 5d-RFSS. We also compared the efficacy of $5 d$-RFSS and CSDS, a validated model of depression (Kudryavtseva et al., 1991; Krishnan et al., 2007; Nestler and Hyman, 2010; Golden et al., 2011), to induce a depressive-like state in C57BL/6J mice.

Predictive validity relies on the observation that treatment modalities effective in reversing depression in humans should reverse the changes observed in an animal model of depression (McKinney and Bunney, 1969; Belzung and Lemoine, 2011). In line with this, several studies have demonstrated that known antidepressant drugs (imipramine, ketamine, fluoxetine, tranylcypromine) or treatments [repetitive transcranial magnetic stimulation (rTMS)] decrease the persistence of increased floating behavior induced by $5 d$-RFSS (Stone and Lin, 2011; Sun et al., 2011; Serchov et al., 2015), thus suggesting pre- 


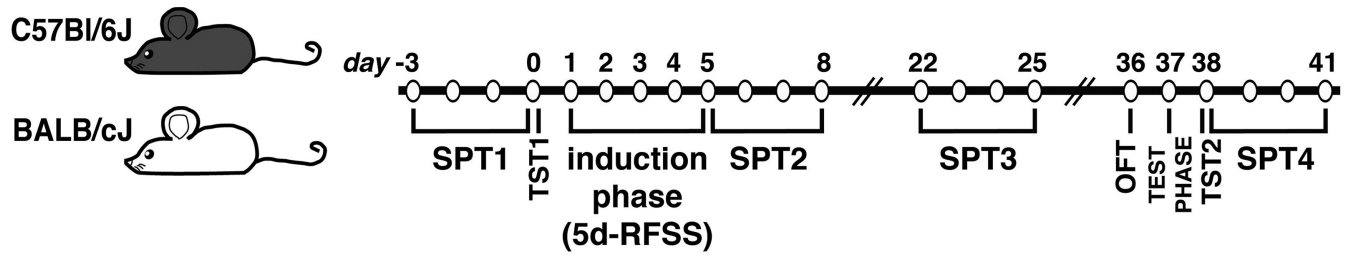

Figure 1. 5d-RFSS paradigm. Experimental timeline of 5d-RFSS in C57BL/6J or BALB/cJ mice. SPT1 and TST1 were performed on days -3 to 0 , before stressed mice underwent 10 min of forced-swim stress during 5 consecutive days (days 1-5; induction phase), followed by SPT2 (days 5-8), SPT3 (days 22-25), an OFT (day 36), the last forced swim session (test phase; day 37), TST2 (day 38), and SPT4 (days $38-41)$. Nonstressed controls were not forced to swim during the induction phase but otherwise underwent the same protocol.

dictive validity. Therefore, we also investigated whether voluntary wheel running (VWR), a behavioral intervention that mimics human exercise training and has antidepressant action (North et al., 1990; Brené et al., 2007), modulates the persistence of increased floating behavior induced by $5 \mathrm{~d}-\mathrm{RFSS}$.

\section{Material and Methods}

\section{Animals}

Experiments were conducted in accordance with the Joslin Diabetes Center Institutional Animal Care and Use Committee. Ten-week old male C57BL/6J (https://www. jax.org/strain/000664) and BALB/cJ (https://www.jax. org/strain/000651) mice were group housed and habituated to the Joslin Diabetes Center animal facility for at least $2 \mathrm{~d}$ before the onset of experimental treatments. This time frame was sufficient for body weights to return to stable pretravel levels (data not shown). Mice were maintained at $23-25^{\circ} \mathrm{C}$ on a $12 \mathrm{~h}$ light/dark cycle (lights on from 6:30 A.M.) with ad libitum access to a pelleted chow diet [9F 5020 Lab Diet (23\% protein, $55 \%$ carbohydrate, and $22 \%$ fat, $3.56 \mathrm{kcal} / \mathrm{g}$ ), PharmaServ] and water, unless noted otherwise. All experimental groups were body weight matched at the onset of the experiments. Body weight and available food were measured at indicated time points. All behavioral assessments, except for the two-bottle sucrose preference tests (SPTs), were performed in an experimental room, and mice were acclimatized to the experimental room for $2 \mathrm{~h}$ before the start of behavioral studies.

\section{The $5 \mathrm{~d}$ repeated forced-swim stress paradigm}

All experimental mice were single housed from the start of the 5d-RFSS (Fig. 1) paradigm. We used a recently described 5d-RFSS protocol (Serchov et al., 2015) with one modification: addition of an extra SPT (i.e., SPT3) during days 22-25. Stressed mice were forced to swim in an open cylindrical container (diameter, $12 \mathrm{~cm}$; height, 28 $\mathrm{cm})$ containing $19 \mathrm{~cm}$ of water $\left(25 \pm 1^{\circ} \mathrm{C}\right)$ on 5 consecutive days (days 1-5; induction phase) and on day 37 (test phase; Fig. 1). Individual tests lasting $10 \mathrm{~min}$ were monitored from the top and scored automatically using the ANY-maze software (version 4.98; Stoelting). Immobile behavior sensitivity was set at $65 \%$, and the mouse needed to be immobile for $500 \mathrm{~ms}$ to initiate the scoring of immobility. In general, each mouse was judged to be immobile when it ceased struggling and remained floating motionless in the water, making only movements necessary to keep its head above the water surface (Duman et al., 2008; Cunha et al., 2013). Water was changed between each test. Nonstressed controls were physically handled briefly by the investigator, transferred to a transport cup for $10 \mathrm{~min}$, and returned to their home cage.

\section{SPT}

Mice were given free access to two drinking pipettes in their home cage, one containing $1 \%$ (C57BL6/J) or 3\% (BALB/cJ) sucrose solution and the other containing water. Fluid consumption was measured in the early afternoon, and the position of the pipettes was interchanged daily to prevent a place preference. Sucrose preference is calculated as the percentage of the amount of sucrose solution consumed over total fluid consumption ([sucrose solution intake/total fluid intake] $\times 100$ ) and was averaged over all $3 \mathrm{~d}$ of testing.

\section{TST}

Mice were suspended by adhesive tape placed $\sim 1 \mathrm{~cm}$ from the tip of their tail that was taped to a horizontal holder so that the mouse was suspended $20 \mathrm{~cm}$ above a horizontal surface. The mouse tail was passed through a small plastic cylinder prior to suspension to prevent tailclimbing behavior. Individual tests lasting $6 \mathrm{~min}$ were monitored and scored automatically using the ANY-maze software (version 4.98; Stoelting). Immobile behavior sensitivity was set at $70 \%$, and the mouse needed to be immobile for $1 \mathrm{~s}$ to initiate scoring of immobility. After the TST, mice were returned to their respective home cages.

\section{Open-field test}

During the light phase, mice were placed in a rectangular open field monitoring setup $(59 \times 29 \mathrm{~cm}$; walls, 30 $\mathrm{cm}$; nonreflecting gray PVC), and locomotor activity was monitored from the top for 6 min and scored automatically using the ANY-maze software (version 4.98; Stoelting). The center zone was defined as a $20 \times 10 \mathrm{~cm}$ zone designated in the middle of the open field. Feces produced during the open-field test (OFT) were counted manually. Mice were immediately returned to their respective home cages after the OFT.

\section{Chronic social defeat stress and behavioral evaluations}

CSDS was performed as described with a few modifications (Krishnan et al., 2007; Vialou et al., 2010; Golden et al., 2011). In short, all mice were tested during SPT1 (day -3 to 0 ) before the start of 10 consecutive days of CSDS. During each defeat episode, experimental 
A

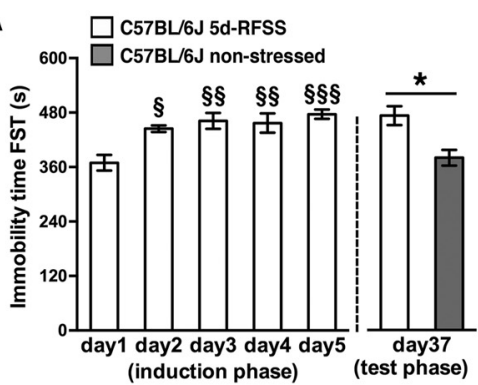

B

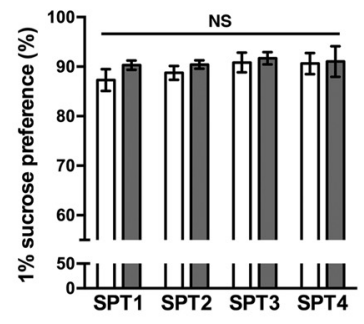

E

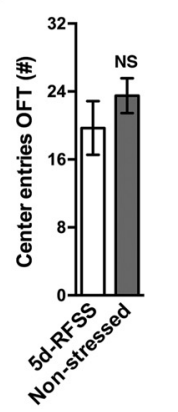

$\mathbf{F}$

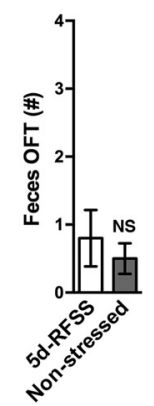

G

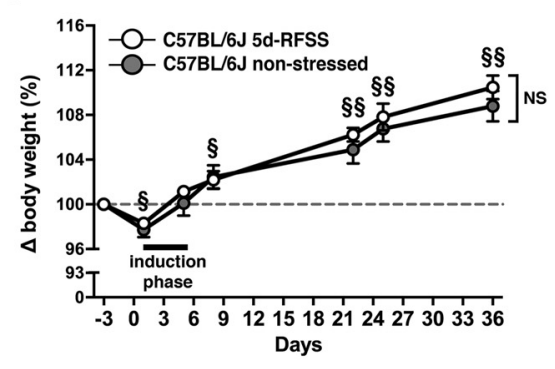

C

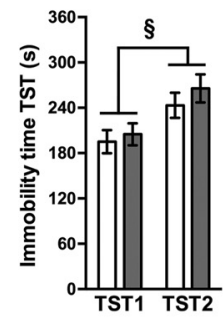

H

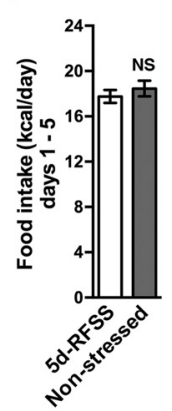

D
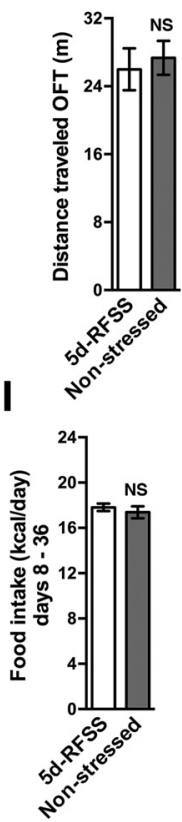

Figure 2. Effects of $5 \mathrm{~d}$-RFSS on depressive-like behavior in C57BL/6J mice. $\boldsymbol{A}$, Immobility time of 5d-RFSS C57BL/6J mice during the induction phase (days 1-5) forced-swim tests (FST; main effect of time, $F_{(4,36)}=8.68, p=0.00005$; post hoc test, $\S p=0.006$, $\S \S p=0.001$, $\S \S \S p=0.0002$ vs day 1$)$, and of $5 d$-RFSS and nonstressed control mice during the test phase (day $37 ; t_{(1,18)}=3.44$, $* p=0.003$ vs nonstressed mice). $\boldsymbol{B}$, Two-bottle SPTs before $5 \mathrm{~d}-\mathrm{RFSS}$ (SPT1) and after $5 \mathrm{~d}-\mathrm{RFSS}$ (SPT2 to SPT4). $\boldsymbol{C}$, TST immobility time before 5d-RFSS (TST1) and after $5 d$-RFSS (TST2; main effect of time, $F_{(1,18)}=9.14, p=0.007$; post hoc test, $\$ p=0.007$ vs TST1). $\boldsymbol{D}-\boldsymbol{F}$, Distance traveled $\left(t_{(1,18)}=0.43, p=0.67 ; \boldsymbol{D}\right)$, number of center zone entries $\left(t_{(1,18)}=1.01, p=0.33 ; \boldsymbol{E}\right)$, and number of feces produced during the OFT $\left(t_{(1,18)}=0.63, p=0.53 ; \boldsymbol{F}\right)$. $\boldsymbol{G}$, Change in body weight (\%) from day -3 to 36 (main effect of time, $F_{(6,108)}=90.48, p<0.00001$; post hoc test, $\S p=0.02$, $\S \S p=0.0001$ vs day -3$)$. $\boldsymbol{H}, \boldsymbol{I}$, Food intake during induction phase (days $1-5$; $\left.t_{(1,17)}=0.77, p=0.45 ; \boldsymbol{H}\right)$ and during days $8-36\left(t_{(1,18)}=0.71, p=0.49 ; \boldsymbol{l}\right)$. NS, Not significant. $n=10 /$ group for all experiments.

C57BL/6J mice (intruder) were allowed to interact for 10 min with an unfamiliar CD1 aggressor (resident), during which they displayed subordinate posturing. Intruders then spent the remainder of each $24 \mathrm{~h}$ period in the cage of the aggressor, separated from the aggressor by a custom-made perforated aluminum partition (sensory housing). Undefeated C57BL/6J controls were housed by pair, one on each side of a perforated aluminum partition (no physical interaction), and were handled daily. For the social interaction test on day 11, time spent in the interaction zone during the first (target absent) and second (social target present) 2.5 min trials were automatically scored using ANY-maze software (version 4.98; Stoelting). Unfamiliar CD1 mice that did not partake in the defeat episodes were used as social targets. Following the social interaction test, all mice were tested during an OFT (day 12) and during SPT2 (days 12-15).

\section{Voluntary wheel running}

Mice were housed without a running wheel (sedentary; SED) or were given voluntary access to an active running wheel (VWR; $24 \mathrm{~cm}$ diameter; Nalgene) for 4 weeks. Wheel revolutions were measured daily using odometers.

\section{Statistical analysis}

Data are displayed as the mean \pm SEM. For all experiments, single comparisons between means were ana- lyzed by unpaired $t$ test, whereas SPT1 and SPT2 were compared using a paired $t$ test. Multiple comparisons between means were analyzed using one- or two-way ANOVA, with repeated measures where applicable. Daily VWR distances were analyzed using one-way ANOVA, with repeated measures. If appropriate, post hoc analyses were made using a Tukey's HSD test, with $p<0.05$ accepted as being statistically different.

\section{Results}

\section{Effects of 5d-RFSS on depressive-like behavior in C57BL/6J mice}

We first tested the hypothesis that $5 d$-RFSS induces a depressive-like state in C57BL/6J mice using a previously described 5d-RFSS protocol (Serchov et al., 2015). In this paradigm, single-housed mice are stressed by being forced to swim on 5 consecutive days (i.e., 5d-RFSS; induction phase) and depressive-like behavior was assessed using a battery of behavioral tests before and after the induction phase (Fig. 1). Nonstressed C57BL/6J controls were handled daily during the induction phase but were not forced to swim. C57BL/6J mice that underwent $5 d-R F S S$ demonstrated a progressive increase in floating behavior during the induction phase (Fig. $2 A$ ). During the test phase $32 \mathrm{~d}$ later, mice that had undergone $5 d$-RFSS continued to demonstrate relatively high levels of floating 
A

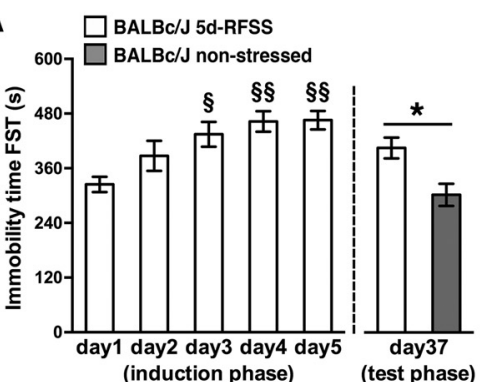

B

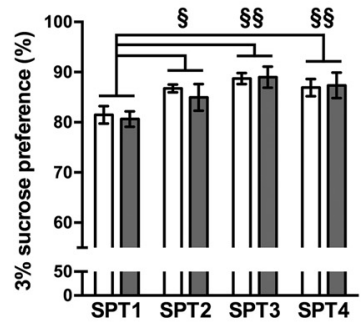

C

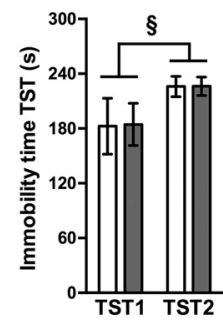

D

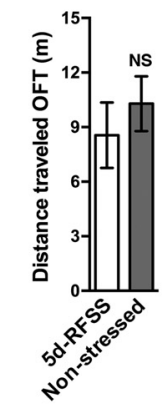

E

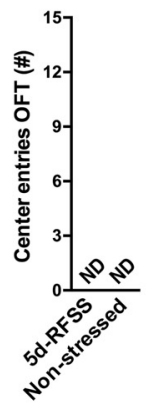

$\mathbf{F}$

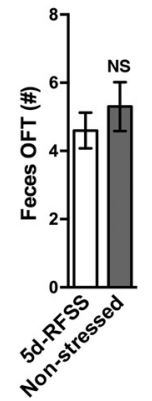

G

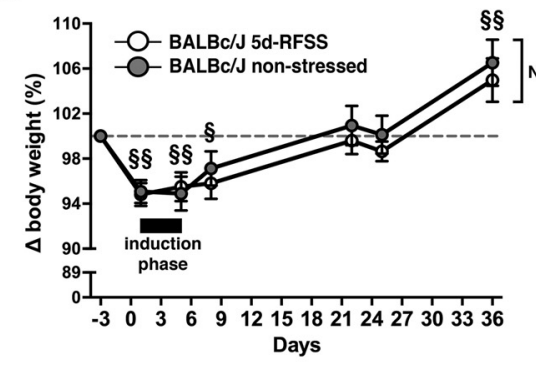

H

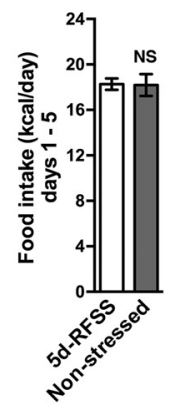

1

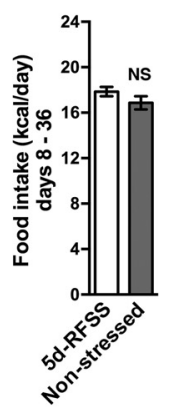

Figure 3. Effects of $5 d$-RFSS on depressive-like behavior in BALB/cJ mice. $\boldsymbol{A}$, Immobility time of $5 d-R F S S$ BALB/cJ mice during the induction phase (days 1-5) of forced swim tests (FST; main effect of time, $F_{(4,36)}=6.29, p=0.0006$; post hoc test, $\S p=0.02$, $\S \S p$ $=0.002$ vs day 1 ) and of $5 d$-RFSS and nonstressed control mice during the test phase (day $37 ; t_{(1,18)}=3.08, * p=0.007$ ). $\boldsymbol{B}$, Two-bottle SPTs before 5d-RFSS (SPT1) and after 5d-RFSS (SPT2 to SPT4; main effect of time, $F_{(3,54)}=12.67, p<0.00001 ;$ post hoc test, $\S p=0.002$, $\S \S p=0.0002$ vs SPT1). $\boldsymbol{C}$, TST immobility time before $5 \mathrm{~d}$-RFSS (TST1) and after $5 \mathrm{~d}$-RFSS (TST2; main effect of time, $F_{(1,18)}=5.06, p=0.04$; post hoc test, $\S p=0.04$ vs TST1). $\boldsymbol{D}-\boldsymbol{F}$, Distance traveled $\left(t_{(1,18)}=0.74, p=0.47 ; \boldsymbol{D}\right)$, number of center zone entries $(\boldsymbol{E})$, and number of feces produced during the OFT $\left(t_{(1,18)}=0.79, p=0.44 ; \boldsymbol{F}\right)$. $\boldsymbol{G}$, Change in body weight $(\%)$ from days -3 to 36 (main effect of time, $F_{(6,108)}=36.96, p<0.00001$; post hoc test, $\S p=0.002$, $\S \S p=0.0001$ vs day -3 ). $\boldsymbol{H}, \boldsymbol{I}$, Food intake during the induction phase (days $\left.1-5 ; t_{(1,18)}=0.07, p=0.94 ; \boldsymbol{H}\right)$ and during days $8-36\left(t_{(1,18)}=1.36, p=0.19 ; \boldsymbol{l}\right)$. NS, Not significant; ND, not detected. $n=10$ group for all experiments.

behavior (Fig. 2A). In line with this persistence, floating behavior scores were significantly greater than those of nonstressed C57BL/6J controls that had not been forced to swim during the induction phase (Fig. 2A). When given a choice between water and $1 \%$ sucrose before undergoing 5d-RFSS (SPT1), C57BL/6J mice showed a typical and strong preference for sucrose ( $~ 88 \%$; Fig. $2 B$ ). None of the $5 d$-RFSS mice developed a loss of sucrose preference (i.e., anhedonia) compared with nonstressed C57BL/6J controls or compared with pre-5d-RFSS (SPT1) levels (Fig. 2B). All experimental mice demonstrated greater immobility scores during TST2 compared with TST1, independent of having been forced to swim (Fig. 2C). Immobility behavior during the test-phase swim session on day 37 or during TST2 was not associated with altered general locomotor activity as indicated by similar distance traveled during an OFT (Fig. 2D). The number of center zone entries or feces produced during the OFT, both of which are parameters indicative of anxiolytic behavior, also did not differ between experimental groups (Fig. 2E,F). Similarly, time spent in the center zone did not differ between nonstressed C57BL/6J controls and mice that underwent the $5 d-R F S S(38.8 \pm 4.7$ vs $34.6 \pm 4.6 \mathrm{~s}$, respectively; $\left.t_{(1,18)}=0.64, p=0.53\right)$. Five consecutive days of forced swimming had no immediate effect on body weight in $5 d$-RFSS mice compared with controls that were handled daily but were not forced to swim (Fig.
2G). All C57BL/6J mice showed similar increases in body weight during the 4 weeks following $5 d$-RFSS, independent of having been forced to swim (Fig. 2G). In contrast, switching from group housing to single housing at the start of the $5 d-R F S S$ paradigm slightly lowered body weight in all C57BL/6J mice (Fig. 2G). 5d-RFSS did not alter caloric intake in the short term during the $5 d-R F S S$ (days 1-5) or in the 4 weeks following 5d-RFSS (days 8-36; Fig. $2 H, I)$.

\section{Effects of $5 d-R F S S$ on depressive-like behavior in BALB/cJ mice}

We next tested the hypothesis that $5 d$-RFSS induces a depressive-like state in BALB/cJ mice. This mouse strain, compared with C57BL/6J mice, is particularly sensitive to the development of stress-induced anhedonia (Farley et al., 2010; Razzoli et al., 2011). Because our preliminary data indicated that BALB/cJ mice do not generate a sucrose preference to a $1 \%$ sucrose solution (data not shown), we used a $3 \%$ sucrose solution with this strain. BALB/cJ mice that underwent $5 d$-RFSS demonstrated a progressive increase in floating behavior during the induction phase (Fig. $3 A$ ). Nonstressed BALB/cJ controls were handled daily during the induction phase but were not forced to swim. During the test phase $32 \mathrm{~d}$ later, mice that had undergone $5 \mathrm{~d}$-RFSS continued to demonstrate increased floating behavior (Fig. $3 A$ ). In line with this per- 
A

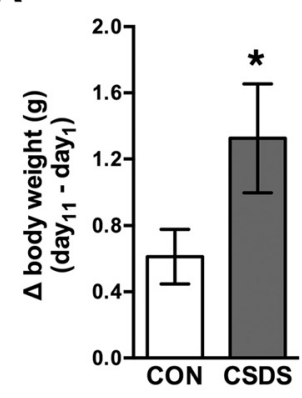

B

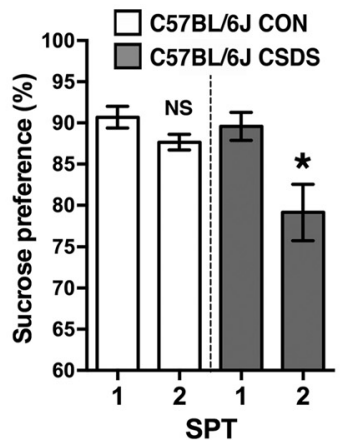

C

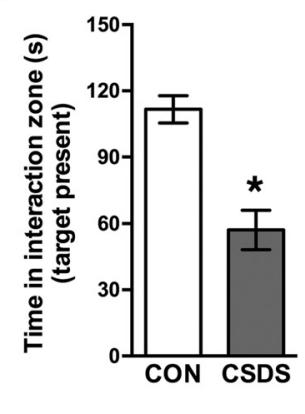

D

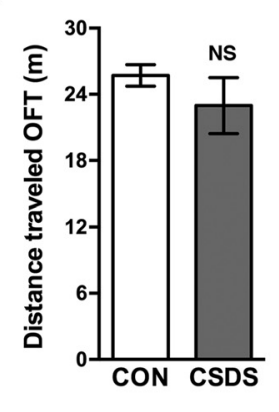

E

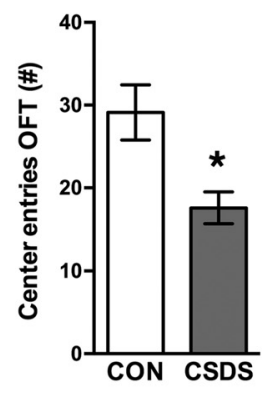

Figure 4. CSDS induces a depressive-like state in C57BL/6J mice. $\boldsymbol{A}$, CSDS mice had greater weight gain compared with nondefeated control (CON) mice (days $\left.1-11 ; t_{(1,14)}=2.28, * p=0.039\right)$. $\boldsymbol{B}$, CON mice did not show a significant decrease in sucrose preference during two-bottle SPT2 compared with SPT1 $\left(t_{(1,7)}=1.96, p=0.1\right)$. CSDS mice demonstrated lower sucrose preference during SPT2 compared with SPT1 (before CSDS; $t_{(1,7)}=3.16, * p=0.016$ ). $\boldsymbol{C}$, CSDS mice spent less time in the interaction zone with target present during the social interaction test on day $11\left(t_{(1,14)}=5.04, * p=0.00018\right)$. $\boldsymbol{D}$, Locomotor activity did not differ significantly between experimental groups during OFT on day $12\left(t_{(1,14)}=1.01, p=0.33\right)$. $\boldsymbol{E}$, CSDS mice enter OFT center zone less than CON mice $\left(t_{(1,14)}=2.99\right.$, $\left.* p=0.01\right)$. NS, Not significant. $n=8 /$ group for all experiments.

sistence, floating behavior scores were significantly greater than those in nonstressed BALB/CJ controls that had not been forced to swim during the induction phase (Fig. 3A). When given a choice between water and $3 \%$ sucrose before $5 d$-RFSS (SPT1), BALB/cJ mice showed a typical and strong preference for sucrose ( $81 \%$; Fig. $3 B$ ). None of the $5 d-R F S S$ mice developed a loss of sucrose preference (i.e., anhedonia) compared with nonstressed BALB/cJ controls or with pre-5d-RFSS (SPT1) levels (Fig. $3 B$ ). Sucrose preference was actually slightly higher during SPT2 to SPT4, independent of the treatment of the mice (Fig. 3B). BALB/CJ mice demonstrated greater immobility scores during TST2 compared with TST1, independent of having been exposed to swim stress (Fig. 3C). Immobility behavior during the test phase swim session on day 37 or during TST2 was not associated with altered general locomotor activity, as indicated by the similar distance traveled during an OFT (Fig. 3D). None of the BALB/cJ mice entered the center zone during the OFT (Fig. 3E). The number of feces produced during the OFT did not differ between 5d-RFSS mice and nonstressed controls (Fig. 3F). Similar to the C57BL/6J cohort, 5 consecutive days of forced swimming had no immediate effect on body weight in 5d-RFSS mice compared with controls that were handled daily but were not forced to swim (Fig. 3G). Furthermore, all BALB/cJ mice showed similar increases in body weight during the 4 weeks following $5 \mathrm{~d}$-RFSS, independent of having been forced to swim (Fig. 3G). In contrast, switching from group housing to single housing at the start of the $5 \mathrm{~d}$-RFSS paradigm slightly lowered body weight in all BALB/cJ mice (Fig. 3G). $5 \mathrm{~d}$-RFSS did not alter caloric intake acutely during $5 \mathrm{~d}$ RFSS (days 1-5) or in the 4 weeks following $5 d$-RFSS (days 8-36; Fig. $3 H, I$ ).

\section{CSDS induces a depressive-like state in C57BL/6J mice}

CSDS is a validated model of depression in C57BL/6J mice (Kudryavtseva et al., 1991; Krishnan et al., 2007; Nestler and Hyman, 2010; Golden et al., 2011). Therefore, we next used this animal model as a comparison to determine our ability to induce a depressive-like state in young-adult male C57BL/6J mice. Mice that had undergone $10 \mathrm{~d}$ of social defeat stress had greater body weight gain compared with nondefeated controls (Fig. 4A). CSDS mice showed a significant decrease in sucrose preference during SPT2 compared with pre-CSDS levels (SPT1), whereas nondefeated controls did not (Fig. 4B). As a second parameter of depressive-like behavior, we also measured social avoidance following CSDS (Krishnan et al., 2007). When tested during a social interaction test on day 11 , CSDS mice spent less time interacting with a social target than nondefeated controls (Fig. 4C). Finally, CSDS mice had fewer center zone entries during an OFT, which is indicative of increased anxiety-like behavior, and this was independent of total locomotor activity during the OFT (Fig. 4D,E).

\section{Effects of VWR on persistence of immobility behavior following $5 \mathrm{~d}-\mathrm{RFSS}$}

Antidepressant drugs and treatments decrease the persistence of increased floating behavior induced by $5 \mathrm{~d}$ RFSS (Stone and Lin, 2011; Sun et al., 2011; Serchov et al., 2015), which suggests predictive validity. Therefore, we tested whether VWR, another known antidepressant intervention (North et al., 1990), could modulate the persistence of immobility behavior induced by $5 d$-RFSS. Following 5d-RFSS, C57BL/6J mice were given voluntary access to running wheels for $28 \mathrm{~d}$ (Fig. 5A,B). VWR after $5 d-R F S S$ lowered the relatively high levels of floating behavior toward nonstressed C57BL/6J control levels (Fig. 5C). In BALB/cJ mice, VWR was even more effective and fully normalized floating behavior to nonstressed BALB/cJ control levels (Fig. 5C,D).

\section{Discussion}

We assessed whether 5d-RFSS has construct, face, and predictive validity to model human depression by determining whether $5 \mathrm{~d}$-RFSS induces a depressive-like state in C57BL/6J and BALB/CJ mice. These inbred 
A

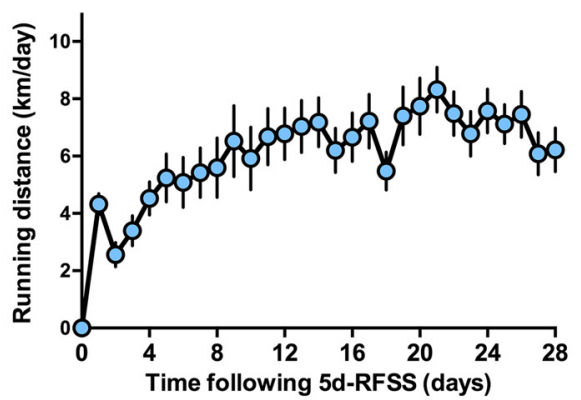

C

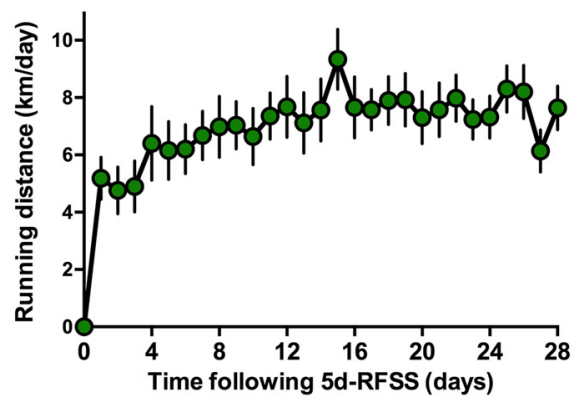

B
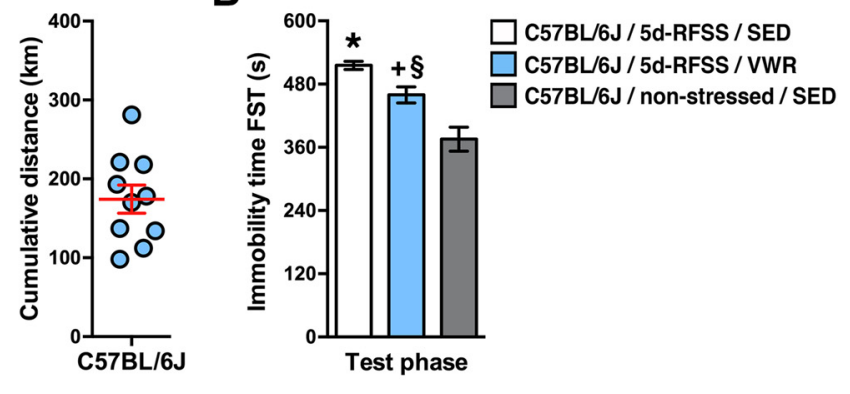

D

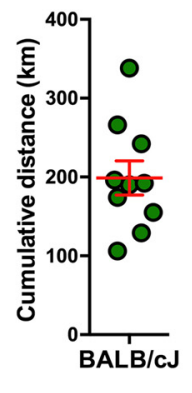

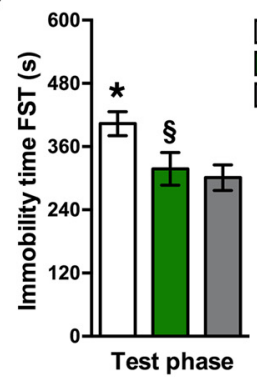

BALBC/J / 5d-RFSS / SED

BALBC/J/5d-RFSS / VWR

BALBC/J / non-stressed / SED

Figure 5. Effects of VWR on the persistence of immobility behavior following 5d-RFSS. $\boldsymbol{A}$, Daily VWR distance (left; $F_{(27,243)}=5.89$, $p<0.00001)$ and cumulative VWR distance (right) of C57BL/6J mice. $\boldsymbol{B}$, VWR (28 d) following $5 \mathrm{~d}-\mathrm{RFSS}$ lowered immobility scores during the test day swim session toward levels of nonstressed SED mice $\left(F_{(2,27)}=18.45, p=0.00001 ; p o s t\right.$ hoc test, $* p=0.00013$ vs nonstressed SED mice; $+p=0.0034$ vs nonstressed SED; $\$ p=0.059$ vs 5d-RFSS SED mice). $\boldsymbol{C}$, Daily VWR distance (left; $F_{(27,243)}$ $=4.37 ; p<0.00001)$ and cumulative VWR distance (right) of BALBc/J mice. $\boldsymbol{D}$, VWR (28 d) following $5 \mathrm{~d}$-RFSS normalized immobility scores during the test day swim session to levels of nonstressed SED mice $\left(F_{(2,27)}=4.41, p=0.02 ;\right.$ post hoc test, *p $=0.026$ vs nonstressed SED, $\S p=0.068$ vs $5 d-R F S S$ SED). $n=10 /$ group for all experiments.

strains, obtained from a commercial breeder, are commonly used in neuropsychiatric research and have relatively low and high emotionality, respectively (Farley et al., 2010; Razzoli et al., 2011). We thoroughly assessed depressive-like behavior before and after $5 \mathrm{~d}$-RFSS by measuring sucrose preference, body weight, food intake, TST immobility behavior, and anxiety-like behavior. Our observations suggest that $5 d-R F S S$ induces an adaptive learned behavioral response, but not a depressive-like state in two mouse strains commonly used in neuropsychiatric research. These findings indicate that $5 d$-RFSS has no construct or face validity to model human depression. However, because the persistence of the adaptive and learned behavioral response is modulated by known antidepressant drugs (Stone and Lin, 2011; Serchov et al., 2015), rTMS (Sun et al., 2011), and VWR (this study), $5 d-R F S S$ might have predictive validity.

In line with previous reports (Sun et al., 2011; Serchov et al., 2015), 5d-RFSS induced a progressive increase in floating during individual swim sessions in our C57BL/6J and BALB/CJ cohorts, and this increase in floating behavior persisted for at least 4 weeks. Our observations thus confirm that repeated forced-swim sessions can induce an adaptive and learned behavioral response underlying survival in mice (Molendijk and de Kloet, 2015).

C57BL/6J or BALB/cJ mice did not develop loss of sucrose preference (i.e., anhedonia) immediately following $5 d$-RFSS or during the 4 weeks following $5 d$-RFSS. In contrast, it has been reported (Serchov et al., 2015) that
$5 d-R F S S$ induced anhedonia immediately after $5 d$-RFSS, and this anhedonia could be rescued following 4 weeks of enhanced adenosine $A_{1}$ receptor expression in the brain. Although experimental differences are potential explanations for the contrasting observations, it is difficult to directly compare our findings, as this study did not specify the exact genetic background, age, or sex of the experimental mice for each individual experiment (Serchov et al., 2015). Our observations suggest that wildtype C57BL/6J and BALB/cJ mice obtained directly from a popular commercial breeder do not develop anhedonia following $5 \mathrm{~d}$-RFSS, suggesting that genetic background can have important effects on behavioral responses to stressors. Finally, a genetic model used by Serchov et al. (2015) was raised on doxycycline in their drinking water until weaning, which, as recognized by the authors, makes water extremely bitter. Thus, alterations in drinking behavior or sucrose-sensing deficits during adulthood cannot be excluded in these studies. Exposure to CSDS has revealed susceptible and unsusceptible subpopulations in C57BL/6J mice (Krishnan et al., 2007). In our 5d-RFSS cohorts, all C57BL/6J and BALB/cJ mice demonstrated stable and high ( $>75 \%)$ sucrose preferences, suggesting that it is unlikely that our experimental cohorts contained susceptible and unsusceptible subpopulations. To properly assess sucrose preference, we averaged drinking behavior over $3 \mathrm{~d}$. Importantly, all mice showed stable consumption behavior during each SPT. Moreover, sucrose preference was stable over the 3 consecutive 
days of SPT1, suggesting that initial reactivity to single housing (conducted on the same day as the start of SPT1) did not contribute significantly.

Changes in body weight or caloric intake are important homeostatic symptoms associated with human depression and can be replicated using established animal models of depression such as CUS and CSDS (Willner, 2005; Nestler and Hyman, 2010). 5d-RFSS did not alter food intake in either strain, both during the $5 d-R F S S$ and in the 4 weeks following the $5 d$-RFSS. Furthermore, 5d-RFSS had no immediate or delayed effect on body weight compared with nonstressed controls in either strain. In contrast, switching from group housing to single housing induced a transient and small decrease in body weight in all mice at the start of the 5d-RFSS protocol. Social isolation can be a mild-to-severe stressor in mice depending on the duration of social isolation (Wallace et al., 2009), suggesting that short-term isolation by single housing of the experimental mice had a larger effect on body weight than $5 d-R F S S$. Body weights were analyzed for 4 weeks following $5 d$-RFSS, suggesting that it is unlikely that body weights will differentiate at a later time point.

All experimental mice demonstrated greater immobility scores during TST2 compared with TST1, again suggesting an adaptive and learned behavioral response. Importantly, TST2 immobility scores were similar between mice that had undergone 5d-RFSS and nonstressed controls. Although the TST is a treatment-based screen with only predictive validity (Nestler and Hyman, 2010), these observations support the notion that $5 d$-RFSS did not induce depressive-like behavior.

Many stress-based rodent models, including CSDS, also exhibit anxiety-like behavior (Krishnan et al., 2007; Nestler and Hyman, 2010). In contrast, 5d-RFSS did not promote anxiety-like behavior compared with nonstressed controls, as indicated by a similar number of center zone entries, center zone time, or feces produced during the OFT. These observations indicate that $5 d-$ RFSS did not induce the anxiety-like behavior often observed in validated rodent models of depression. BALB/cJ mice produced more feces and showed complete avoidance of the center zone during the OFT compared with C57BL/6J mice, confirming their greater emotionality (Farley et al., 2010; Razzoli et al., 2011).

Together, our observations indicate that $5 d$-RFSS did not induce emotional, homeostatic, or psychomotor symptoms in young-adult male C57BL/6J and BALB/cJ mice obtained from a commercial breeder. In contrast, CSDS induced changes in body weight, a significant decrease in sucrose preference (i.e., anhedonia), social avoidance, and increased anxiety-like behavior in youngadult male C57BL/6J mice. Collectively, these symptoms are indicative of a depressive-like state (Krishnan et al., 2007; Nestler and Hyman, 2010). Although we cannot exclude that $5 d$-RFSS can induce a depressive-like state in older mice or in stress-susceptible genetic models, our findings suggest that $5 d$-RFSS has no construct or face validity to model human depression in two inbred strains commonly used in neuropsychiatric research. However, because known antidepressant treatments, including drugs (Stone and Lin, 2011; Serchov et al., 2015), rTMS (Sun et al., 2011), and VWR (this study), can modulate the persistence of increased floating behavior, 5d-RFSS might have predictive validity to identify novel antidepressant treatments. Finally, the initial assessment of depressive-like behavior in rodents can include treatment-based screens, such as the forced-swim test and TST. However, a combination of emotional, homeostatic, and psychomotor symptoms should be measured to provide more definitive evidence of a depressive-like state.

\section{References}

Belzung C, Lemoine M (2011) Criteria of validity for animal models of psychiatric disorders: focus on anxiety disorders and depression. Biol Mood Anxiety Disord 1:9. CrossRef Medline

Brené S, Bjørnebekk A, Aberg E, Mathé AA, Olson L, Werme M (2007) Running is rewarding and antidepressive. Physiol Behav 92:136-140. CrossRef Medline

Cunha MP, Oliveira Á, Pazini FL, Machado DG, Bettio LE, Budni J, Aguiar AS Jr, Martins DF, Santos AR, Rodrigues AL (2013) The antidepressant-like effect of physical activity on a voluntary running wheel. Med Sci Sports Exerc 45:851-859. CrossRef

Duman CH, Schlesinger L, Russell DS, Duman RS (2008) Voluntary exercise produces antidepressant and anxiolytic behavioral effects in mice. Brain Res 1199:148-158. CrossRef Medline

Farley S, Apazoglou K, Witkin JM, Giros B, Tzavara ET (2010) Antidepressant-like effects of an AMPA receptor potentiator under a chronic mild stress paradigm. Int $\mathrm{J}$ Neuropsychopharmacol 13:1207-1218. CrossRef Medline

Golden SA, Covington HE 3rd, Berton O, Russo SJ (2011) A standardized protocol for repeated social defeat stress in mice. Nat Protoc 6:1183-1191. CrossRef

Greenberg PE, Kessler RC, Birnbaum HG, Leong SA, Lowe SW, Berglund PA, Corey-Lisle PK (2003) The economic burden of depression in the United States: how did it change between 1990 and 2000?. J Clin Psychiatry 64:1465-1475. Medline

Kessler RC, Berglund P, Demler O, Jin R, Merikangas KR, Walters EE (2005) Lifetime prevalence and age-of-onset distributions of DSM-IV disorders in the National Comorbidity Survey Replication. Arch Gen Psychiatry 62:593-602. CrossRef Medline

Krishnan V, Nestler EJ (2008) The molecular neurobiology of depression. Nature 455:894-902. CrossRef Medline

Krishnan V, Han MH, Graham DL, Berton O, Renthal W, Russo SJ, Laplant Q, Graham A, Lutter M, Lagace DC, Ghose S, Reister R, Tannous P, Green TA, Neve RL, Chakravarty S, Kumar A, Eisch AJ, Self DW, Lee FS, et al. (2007) Molecular adaptations underlying susceptibility and resistance to social defeat in brain reward regions. Cell 131:391-404. CrossRef Medline

Kudryavtseva NN, Bakshtanoversuskaya IV, Koryakina LA (1991) Social model of depression in mice of C57BL/6J strain. Pharmacol Biochem Behav 38:315-320. Medline

McKinney WT Jr, Bunney WE Jr (1969) Animal model of depression. I. Review of evidence: implications for research. Arch Gen Psychiatry 21:240-248. Medline

Molendijk ML, de Kloet ER (2015) Immobility in the forced swim test is adaptive and does not reflect depression. Psychoneuroendocrinology 62:389-391. CrossRef Medline

Nestler EJ, Hyman SE (2010) Animal models of neuropsychiatric disorders. Nat Neurosci 13:1161-1169. CrossRef Medline

North TC, McCullagh P, Tran ZV (1990) Effect of exercise on depression. Exerc Sport Sci Rev 18:379-415. Medline

Pothion S, Bizot JC, Trovero F, Belzung C (2004) Strain differences in sucrose preference and in the consequences of unpredictable chronic mild stress. Behav Brain Res 155:135-146. CrossRef Medline 
Razzoli M, Carboni L, Andreoli M, Ballottari A, Arban R (2011) Different susceptibility to social defeat stress of BalbC and C57BL6/J mice. Behav Brain Res 216:100-108. CrossRef Medline

Serchov T, Clement HW, Schwarz MK, lasevoli F, Tosh DK, Idzko M, Jacobson KA, de Bartolomeis A, Normann C, Biber K, van Calker D (2015) Increased signaling via adenosine A1 receptors, sleep deprivation, imipramine, and ketamine inhibit depressive-like behavior via induction of Homer1a. Neuron 87:549-562. CrossRef Medline

Stone EA, Lin Y (2011) Open-space forced swim model of depression for mice. Curr Protoc Neurosci Chapter 9:Unit9.36.

Sun $P$, Wang F, Wang L, Zhang $Y$, Yamamoto R, Sugai T, Zhang Q, Wang Z, Kato N (2011) Increase in cortical pyramidal cell excitability accompanies depression-like behavior in mice: a transcranial magnetic stimulation study. J Neurosci 31:16464-16472. CrossRef Medline
Vialou V, Robison AJ, Laplant QC, Covington HE 3rd, Dietz DM, Ohnishi YN, Mouzon E, Rush AJ 3rd, Watts EL, Wallace DL, Iñiguez SD, Ohnishi YH, Steiner MA, Warren BL, Krishnan V, Bolaños CA, Neve RL, Ghose S, Berton O, Tamminga CA, et al. (2010) DeltaFosB in brain reward circuits mediates resilience to stress and antidepressant responses. Nat Neurosci 13:745-752. CrossRef Medline

Wallace DL, Han MH, Graham DL, Green TA, Vialou V, Iñiguez SD, Cao JL, Kirk A, Chakravarty S, Kumar A, Krishnan V, Neve RL, Cooper DC, Bolaños CA, Barrot M, McClung CA, Nestler EJ (2009) CREB regulation of nucleus accumbens excitability mediates social isolation-induced behavioral deficits. Nat Neurosci 12:200209. CrossRef Medline

Willner P (2005) Chronic mild stress (CMS) revisited: consistency and behavioural-neurobiological concordance in the effects of CMS. Neuropsychobiology 52:90-110. CrossRef Medline 